\title{
PHOTONIC ARCHITECTURES IN BEETLES: TWISTS AND IRIDESCENCE
}

\author{
L.T. MCDONALD, T.A. STARKEY \& P. VUKUSIC \\ School of Physics, University of Exeter, UK.
}

\begin{abstract}
The order Coleoptera is, by any standard, a prodigious showcase for the extraordinary creativity and flexibility of the evolutionary process. Concurrent with a desire to overcome the present limitations of optical coating technologies, a number of novel and elegant reflectance mechanisms have been discovered in the realms of biological systems including 3D photonic crystals and quasi-ordered coherent scattering arrays. Beetles, in particular, possess many desirable and, crucially, tunable properties from a biomimetic perspective. Here, we provide a detailed discussion of two coleopteran structures, namely 1D multilayers and helically arranged 'Bouligand' structures, and consider their putative entomological functions and potential applications in bioinspired technologies.

Keywords: Beetles, biomimetics, broadband, circular polarisation, iridescence, multilayer, optical activity, structural colour.
\end{abstract}

\section{INTRODUCTION}

Biological systems primarily use three processes to generate colour appearance, namely, through wavelength-selective absorption by pigment, luminescence processes, or through coherent scattering by sub-wavelength structures. Nature's use of pigmentation in providing visual stimuli is commonplace and well understood, with chemical-based light processes controlled by chromophores in the organism [1]. Many species of flora and fauna synthesise pigments including melanins, ommochromes and papiliochromes. Colours produced via pigmentation are often relatively broadband and display low-intensity, diffuse, angle-independent hues. By contrast, colours that originate through the coherent scattering of light by a structure, with features on the micro- and nanoscale, are vibrant and enduring. Recently, structural colouration has become a burgeoning research topic for biologists and physicists alike [2,3] and has been reported in insect, avian and marine phyla as well as several plant species [4-6].

The world of beetles provides the inquisitive mind with a tapestry richly diverse in colour-producing architectures for scientific study [7]. In allusion to their striking visual aesthetics, beetles have been christened 'living jewels'. This paper offers a detailed description of the role of 1D photonic crystal, or 'multilayer', structures in Coleoptera. The layered architectures found in beetles can be broadly considered using two distinctly different structural models. The first, and most commonly observed, is a multilayer structure that comprises strata with alternating high and low refractive indices (RIs). Second, the optical phenomena of some species directly relates to their 'twisted', chitin-rich multilayer structure. This second architecture is optically active, i.e. the polarisation plane of light is altered as it propagates through the beetle's cuticle, resulting in a circularly polarised reflection. The cuticle parameters effective in tailoring the optical response from these structures are presented and discussed with respect to their potential applications in the advancing field of biomimetics and bioinspired technology. 


\section{A BRIEF NOTE ON CUTICLE FORMATION AND MICROSTRUCTURE}

The cuticle of beetles is a sophisticated integumentary system constructed layer by layer from materials segregated by underlying epidermal cells [8]. As individual layers are modified through stages of growth both chemical composition and optical properties develop (the specifics of which are documented in detail elsewhere [8,9]). Chitin is recognised to be the dominant component in the composite structure that contains other materials, including proteins, melanin and lipids. The cuticle comprises three main sections working downwards from the surface: the epicuticle, the exocuticle and the endocuticle. The beetles' optical properties are largely controlled by the epi- and exocuticle regions.

Both the epicuticle and the exocuticle undergo sclerotisation (hardening) and tanning processes after formation to fix their stiffness and chemical stability leaving them with an opaque brownish colour. The epicuticle is typically $1-2 \mu \mathrm{m}$ thick. This is relatively thin compared with the exocuticle, which can be as thick as $100 \mu \mathrm{m}$. Imaging cross-sections of the elytra, using both scanning electron microscopy (SEM) and transmission electron microscopy (TEM) techniques, is the most direct method for quantitatively ascertaining the dimensional specifics of the cuticle.

\section{ONE-DIMENSIONAL PHOTONIC CRYSTALS}

Although they have been studied in one form or another for over a century, photonic crystals (PhCs) are a relatively modern concept in the field of photonics. The theory, conceived following the works of Yablonovitch and John in the late 1980s [10,11], centres around the analogy that photons propagating through a periodic dielectric structure are akin to the motion of electrons in a semiconductor crystal, whereby a periodic potential defines the presence and position of band gaps in the structure. $\mathrm{PhCs}$, therefore, affect the propagation of electromagnetic waves within a specific frequency range. By arranging periodically varying RIs in one, two or in all three spatial directions, opportunities arise to bend, slow and tailor the propagation of light [12]. Whilst only 1D structures are outlined in this paper, many biological systems have evolved 2D and 3D RI periodicities in order to confer precise visual appearances that underpin particular biological functions.

Since the theory of $\mathrm{PhCs}$ is described extensively elsewhere [12], here one important physical consequence of light incident on a PhC is highlighted, namely, the photonic band gap (PBG). When light is incident on a medium with periodically changing RIs, for instance a 1D system comprising high and low RI layers, it may be transmitted or reflected to different extents. Light incident with a wavelength satisfying the resonant conditions of a 1D system, as dictated by the periodicity and RIs of the layers, creates standing waves at two different energies. Their presence results in a region in which electromagnetic radiation incident on the $1 \mathrm{D} \mathrm{PhC}$ is forbidden from propagating. The size of this region is strongly influenced by the constituent RIs of the PhC. In the case of a photonic solid with finite layers, the PBG is usually determined as the wavelength regions at which no incident radiation may propagate. Obtaining a full and complete photonic band gap (FCPBG), where light is prohibited from propagating from all incident angles upon the crystal, is one of the most pressing challenges when fabricating synthetic $\mathrm{PhC}$ structures.

\subsection{Multilayer interference}

Beetles commonly exhibit vivid, metallic colours achieved using multiple thin films, or multilayer systems. Here, peak reflectance can be determined using the same geometric considerations as those for single thin films [13].

Consider multilayers comprising pairs of thin films (Fig. 1a); layer 1 has RI equal to $n_{1}$ and thickness $d_{1}$; layer 2 has RI equal to $n_{2}$ and thickness $d_{2}$. Light is incident at an angle $\theta_{0}$ from a medium with RI $n_{0}$. The condition for constructive interference is: 


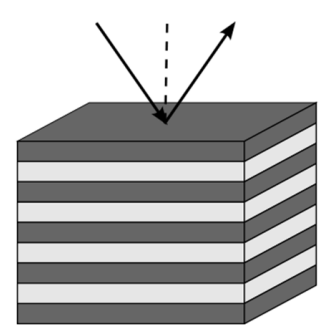

(a)

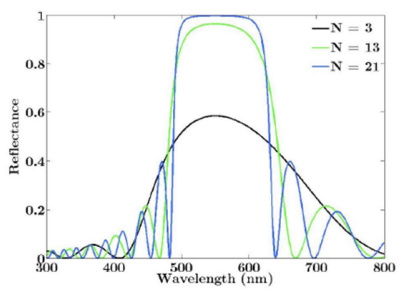

(b)

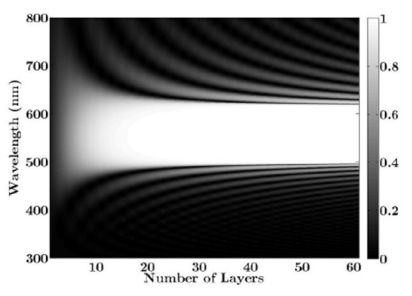

(c)

Figure 1: (a) A schematic for a generic multilayer structure; (b) the reflectance at normal incidence, $R_{\text {norm }}$, of an ideal multilayer; and (c) a reflectance map showing the theoretical variation of $R_{\text {norm }}$ with increasing $N$.

$$
m \lambda=2\left(d_{1} \sqrt{n_{1}^{2}-n_{0}^{2} \sin ^{2} \theta_{0}}+d_{2} \sqrt{n_{2}^{2}-n_{0}^{2} \sin ^{2} \theta_{0}}\right)
$$

where $m$ is an integer. When the optical thickness of both layers is equal, the system is referred to as an ideal multilayer [14], and for normal incidence the interference condition reduces to

$$
\frac{\lambda}{4}=n_{1} d_{1}=n_{2} d_{2}
$$

Ideal multilayers are a special case, however. Although they are found in some biological examples, such as some fish species' scales [15], the majority of biological multilayers are non-ideal.

\subsection{Factors affecting reflectance in biological systems}

The reflectance of a 1D PhC structure composed of two different materials, with RIs $n_{1}$ and $n_{2}$, respectively, has several key influences: the number of layers $N$, the RI contrast $\hat{n}=n_{1} / n_{2}$ (assuming $n_{1}>n_{2}$ ), the polarisation state and the angle $\theta_{0}$ of incident light, the thickness of the individual layers $d_{1}, d_{2}$ and the presence of optical absorption. The influence of these parameters on the reflectivity of ideal multilayer quarter-wave stacks is presented (Figs. 1 and 2) using standard methods [13]. The models assume air as the incident and exit media. In biological systems, these variables can be set or controlled by beetles' morphology, behaviour or local ecology.

With increasing $N$, the reflectance intensity increases while the bandwidth of the reflection peak decreases (Fig. $1 \mathrm{~b}$ and c). At light incidence angles $\theta_{0} \neq 0^{\circ}$, the increase in reflectivity is more pronounced for perpendicularly polarised light. For small enough layer numbers, there is a significant difference between $R_{\perp}$ and $R_{\|}$and consequently unpolarised light becomes partly polarised upon reflection from a multilayer; this difference vanishes for large $N$. Species with a high $N$ tend to display more intense colours and often appear metallic if the layers are well aligned. In spite of this, however, examples of biological systems comprising more than 20 layers are uncommon [16]. Similarly, increasing $\hat{n}$ also leads to an increase in reflectivity (Fig. 2c). Again, the increase is more pronounced for perpendicularly polarised light with the difference between $R_{\perp}$ and $R_{\|}$vanishing at higher contrasts. While, in technology, photonic systems may be designed and fabricated using a range of high-index materials, biological processes are largely limited to the use of simple dielectric materials. RI values rarely exceed 1.8 , with $\hat{n}$ rarely exceeding 1.56 . Although these values do not prevent bright colours being created, they do limit the extent to which a complete PBG can be established. For increasing angles of light incidence $R_{\perp}$ increases monotonically towards unity while $R_{\|}$ 


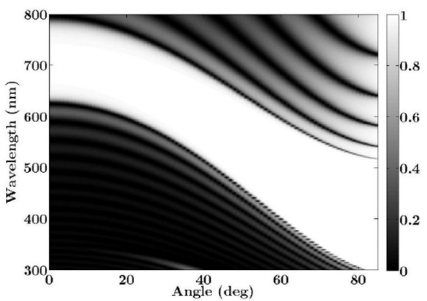

(a)

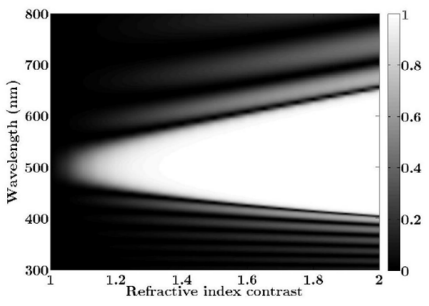

(c)

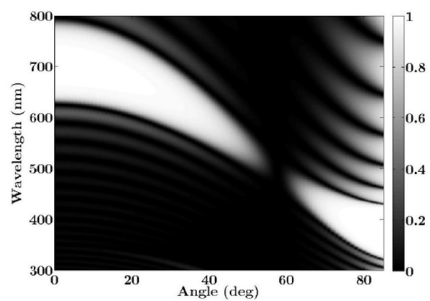

(b)

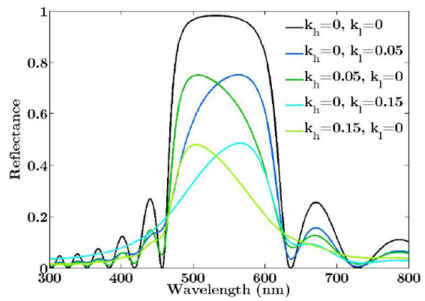

(d)

Figure 2: Reflectance maps showing: (a) and (b) the angle-dependence of $R_{\perp}$ and $R_{\|}$, respectively, and the theoretical dependence of $R_{\text {norm }}$ on (c) $\hat{n}$ and (d) $k$ for an ideal multilayer ( $k_{\mathrm{h}}$ and $k_{1}$ denotes high and low RI layers, respectively).

first decreases, reaching a minimum at the generalised Brewster angle $\theta_{\mathrm{B}}$ of the multilayer stack, before it increases again (Fig. $2 a$ and $b$ ).

The RI index of the most materials is a complex value comprising both the real part $n$ and the imaginary part $k$. Optical absorption in the system is governed by $k$; increasing $k$ results in the intensity of the reflectance at the peak wavelength $\lambda_{\max }$ decreasing. When absorption is present in the high RI layers, there is a large attenuation of the high-wavelength band-edge, with the opposite being true when absorption is present in the low RI layers (Fig. 2d). In general, a multilayer structure has to be tailored to its specific purpose, such as controlling the position and intensity of $\lambda_{\max }$, by using appropriate materials and by suitably adapting the film thicknesses.

\subsection{Biological multilayers in Frog-leg beetles}

Species belonging to the genus Sagra are recognisable by their hind legs, which are far longer and stronger relative to the front two pairs. In addition, certain species such as Sagra buqueti, featured here, exhibit striking sexual dimorphism.

TEM images reveal the underlying structures responsible for the iridescent colours observed in the elytra of $S$. buqueti (Fig. 3a) and, to a lesser extent $S$. femoralis (Fig. 3b). TEMs taken from the blue-green outer region of $S$. buqueti's elytron, and the majority of $S$. femoralis, show a uniform $1 \mathrm{D}$ multilayer with an average of 16 layers differing only in thickness (Fig. 3d and f). The inside edge of $S$. buqueti's wing, which displays rich hues ranging from persimmon to magenta, has a consistent set of two layers at the top of the 11-12 layer stack separated from deeper layers by a larger fifth layer (Fig. 3c and e). Layer thicknesses range from 50 to $200 \mathrm{~nm}$. Electron dense layers, which comprise a melanoprotein material, appear as darker in the TEM images and have RI $n=1.68$, whilst the lighter, electron lucent, chitin layers have a RI $n=1.56$. Optical absorption is negligible in the chitin layers $(k=0.03)$ but its presence in the melanoprotein layers $(k=0.14)$ noticeably influences the 

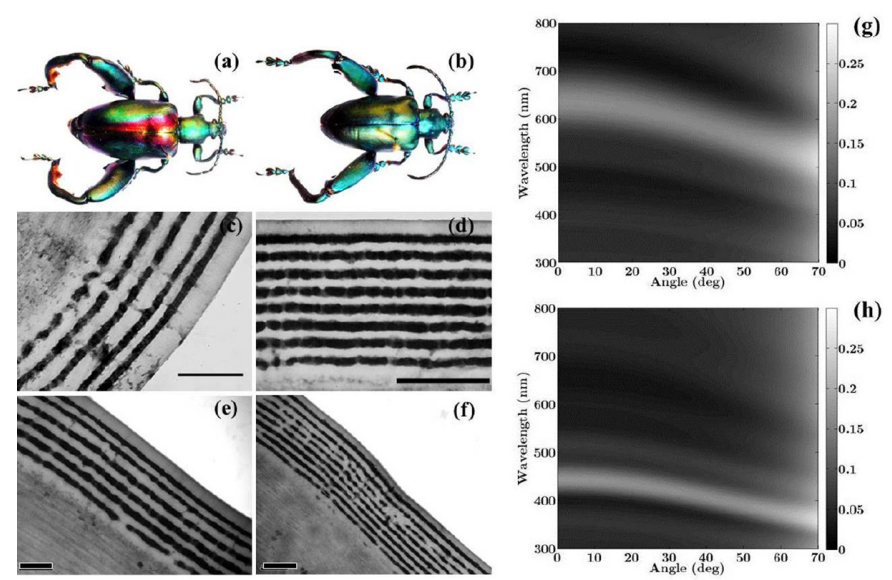

Figure 3: (a) S. buqueti and (b) S. femoralis with their respective TEM cross-sections and modelled angle-dependent reflectance. [Scale bars are (c, e, f) $0.5 \mu \mathrm{m}$ and (d) $1 \mu \mathrm{m}$ ].

structure's appearance. Owing to optical dispersion in both materials, with the magnitude of both $n$ and $k$ increasing for shorter wavelengths, these values are averages; they are typical of those found in beetles, and also in other biological systems besides [17]. The TEM images (Fig. 3b-f) show that the structure controlling the reflectance is located in the beetles' epicuticle. Parameters obtained from the images were used to model the red-orange reflecting region in S. buqueti (Fig. $3 \mathrm{~g}$ ) and the blue-green reflecting multilayer in $S$. femoralis (Fig. $3 \mathrm{~h}$ ).

\subsection{Optical activity in beetle cuticle}

In a twist upon the one-dimensional multilayer reflector, some beetles exhibit multilayer structures able to selectively reflect circularly polarised (CP) light (Fig. 4). This ability is dictated by a helical deposition of chitin microfibrils to form the so-called 'Bouligand' structures in which long threadlike chiral molecules form to give the stratified architecture. This arrangement is analogous to cholesteric liquid crystals; within individual layers all threads lie parallel, but in moving from one layer to the next, the orientation of the threads is rotated by a small and constant angle (Fig. 4d). The distance over which the threads are rotated through $360^{\circ}$ is the helicoidal pitch. CP light with the same handedness as the helicoids is reflected by the structure whilst light with the opposite handedness is transmitted $[16,18]$.

Most photonic biological systems employ two materials, each with a different RI. An interesting aspect of Bouligand structures is that each layer in the stack is made from the same material; the threads in the layers are birefringent with different values for the RI running parallel and perpendicular to the cuticle surface. Several optical models have previously been described that identify the $R \mathrm{I}_{\perp}$, i.e. in the direction of the thread, is higher than the $\mathrm{RI}_{\|}[18-20]$.

\subsubsection{Helicoid formation and liquid crystal analogy}

The event of CP reflections from scarab beetles was first observed by Michelson in 1911 [21], who postulated that the effect must be determined by a 'screw structure' of ultra-microscopic dimensions. Their optical properties were compared with those of liquid crystals (LCs) formed by cholesterol 

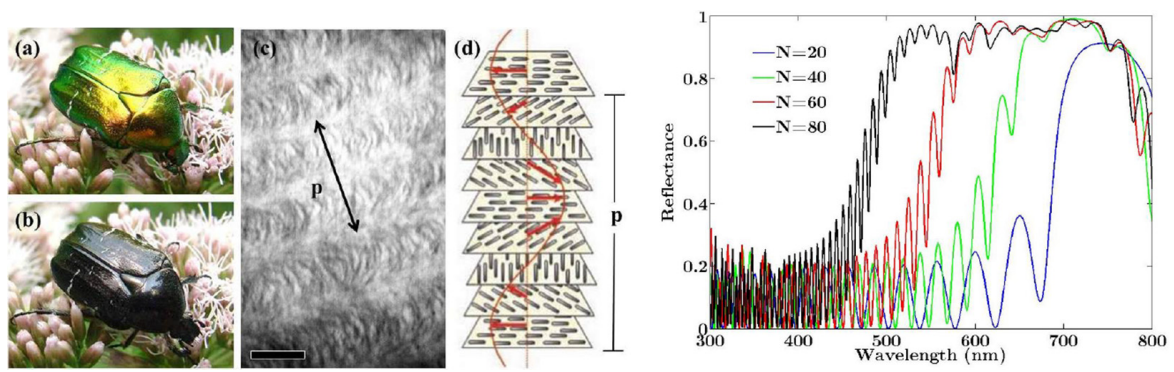

Figure 4: The LCP light reflecting scarab beetle C. aurata viewed through (a) a left-handed and (b) a right-handed CP filter; (c) the arched pattern in TEM images and (d) the relative orientation of sublayers in the chiral structure (reproduced from [20]), with the pitch length $p$ illustrated. Plot (e) illustrates $R_{\text {norm }}$ for a chirped system for different $N$ values.

derivatives as early as the 1920s [16]. However, it required the emergence of electron microscopy techniques for the analogy to be consolidated. TEM images of adult scarab beetles revealed a cuticle constructed of a complex mesh of arcs (Fig. 4c). Bouligand is credited with attributing these arcs to the layered distribution comprising successive molecular orientations. He stressed that the direct relationship between the cholesterics and the Bouligand structure strongly suggests that biological macro-molecules with this organisation first go through a stage as LCs [22].

Many biological nanoarchitectures incorporate these long thread-like molecules of materials such as cellulose, chitin and collagen. As the understanding of the molecular structures has advanced, Bouligand structures have been reported in a variety of biological systems [23-26], implying there is a general principle governing the formation of all these structures.

Experiments with suspensions of collagen indicate that molecules can self-assemble to form Bouligand structures. Cholesteric LCs are characterised by the absence of positional order and by the long-range orientational order of the long molecular axes. In the Bouligand structure, however, the freedom of the individual molecules is restricted. In arthropods, the phase of the cuticle's constituent materials is changed by the action of sclerotisation and tanning.

\subsubsection{Reflection by helicoidal structures}

When white light is incident on the surface of a helicoidal structure, such as cuticle of some scarab subfamilies, selective reflection occurs. If the pitch of the structure is comparable to visible wavelengths, Bragg-like reflection is observed with a maxima at the wavelength given by $\lambda=p n \sin \theta$, where $p$ is the pitch length, $n$ is the RI and $\theta$ is the incident angle [16]. For a given colour, the reflection maximum is observed when the optical thickness of a half-pitch is equal to the wavelength of the colour. Thus, the Bouligand architecture behaves as a half-wave interference system. Where the structure possesses many helical rotations, each pitch relates to reflection at one wavelength and the reflected intensity increases with the number of pitches [27]. Iridescent behaviour is consistent with that of non-optically active multilayer structures. A continuously varying pitch length manifests in a chirped multilayer structure that gives rise the broadband colours of many beetles. Others, such as Chrysina boucardi, employ pitch variance differently to give more narrowband colours [19].

With respect to TEM images of Bouligand structures, when the beetle is viewed through crossed polarisers from any direction perpendicular to the axis of rotation of the 'threads', there will be 

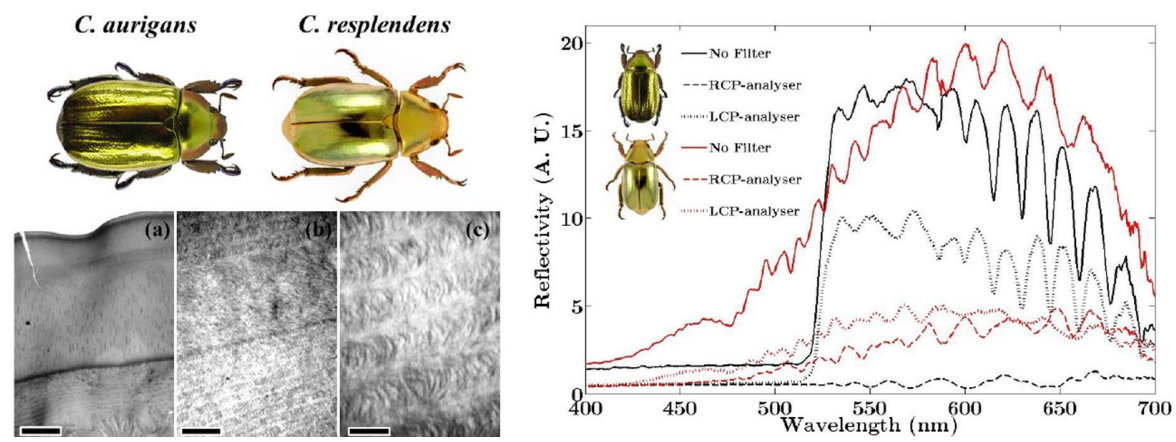

Figure 5: TEM images of Chrysina resplendens (a-c) reveal the HWP layer with the plot (right) providing a comparison of the $\mathrm{CP}$ analysed reflection from Chrysina resplendens with Chrysina aurigans. [Scale bars: (a) $5 \mu \mathrm{m}$; (b) $0.5 \mu \mathrm{m}$ and (c) $0.1 \mu \mathrm{m}$ ].

retardation maxima alternating with retardation minima for every $180^{\circ}$ rotation. The system, therefore, appears to show two lamellae per $360^{\circ}$ rotation (Fig. 4c).

\subsection{Circularly polarising reflectors in the genus Chrysina}

The circularly polarised reflectance intrinsic to helicoidal structures is observed in beetles for several subfamilies of Scarabaeidae, namely Rutelinae, Scarabaeinae and Cetoniinae [28]. Biological systems reflect left-handed CP light (LCP) almost without exception and those belonging to the genus Chrysina are no different, though they include one notable exception. Chrysina resplendens bears the remarkable ability to reflect both LCP and RCP light simultaneously.

Referring to TEMs (Fig. 5a-c) of an oblique incision through the optically active reflecting cuticle provides the explanation. C. resplendens is shown to possess two spatially distinct regions of helicity separated by a relatively thick, non-helicoidal or 'unidirectional' layer. The thickness of this layer is carefully tuned so that it performs the function of a half wave plate (HWP), changing the handedness of light transmitted by the first helicoidal region. Thus, right-handed (RCP) incident light becomes left-handed (LCP) and interacts with the structure in the second helicoidal region. Upon reflection, this light passes back through the HWP layer, is reconverted to RCP light and is transmitted back through the structure without further interaction. The whole construction thus reflects both LCP and RCP light. For all biological species studied so far, the HWP layer is a structural feature unique to C. resplendens; other species such as Chrysina aurigans and Chrysina optima reflect LCP light almost exclusively (Fig. 5). The pitch length varies continuously in species of the Chrysina genus, giving rise to their gold and silver appearances. In the case of $C$. resplendens, $\mathrm{RI}_{\perp}$ and the average $\mathrm{RI}_{\|}$are given as $n=1.701$ and $n=1.603$, respectively [18].

\section{BIOLOGICAL FUNCTION AND BEETLE BIOMIMETICS}

In biological systems, the optical cause and effect that is associated with the capability of periodic structures to manipulate light, thereby giving rise to distinct optical appearances, is now the subject of genuine interdisciplinary scientific interest. Achieving precise understanding of the mechanism by which an exotic-looking animal or plant derives its intense saturated colour, its deep black or brilliant white appearance or possibly even its dramatic linear or circular polarisation signature, can be an eminently exciting and scientifically rewarding process. From the perspectives of evolutionary 
diversity, behavioural biology and general zoology, materials science, mainstream optics and photonics, self-assembly chemistry and bioinspired technology, the study of light and colour manipulation in biological systems is yielding rich reward.

The precise biological function of structural colours, whilst obvious in some species, is more obscure in others. The vibrancy of colours in many species makes them conspicuous by appearance and is not necessarily advantageous. As such, structural colours must be considered in both an ecological and a zoological context. Many iridescent beetles are native to tropical rainforests where the tree canopy fractures sunlight as it passes through to produce narrow and, often, pulsed rays. As a consequence, many beetles use 'flash colouration', whereby the movement between areas of light and dark creates high visual contrast to confuse predators. Furthermore, species bearing metallic elytra are highly reflective providing crypsis with the surrounding foliage. Other visual functions of structural colours include sexual signalling, both salient and covert polarisation effects and aposematic colours. Non-visual purposes, for example, thermoregulatory and friction-reducing mechanisms have also been described [7].

The visual effects manifested in beetle species range from narrowband to broadband reflections determining the perceived colour of the structure's surface. Metallic and diffuse effects are both observed, with the resultant lustre dependent on the directionality of the reflection. The angle dependency of reflected colours is a further demonstration of the diversity of structural mechanisms employed across the order. Pigment-assisted colouration, moreover, is not unusual for tuning colours arising from cuticle structures. Nature's designs also provide a basis for the technical parameters of fabricated reflectors, i.e. layer number and thickness and their organisation. Inspiration for pitch variation and the number of spirals in a helicoid can be drawn from studying the Bouligand structure in scarab species. In certain cases, beetles exhibit cutilcular designs that enable them to control the polarisation and thereby combinations of transparency reflectivity. Further to their aesthetic qualities, beetles thrive in sustainable ecosystems and thus comprise renewable materials that offer comparable visual effects to metals whilst being both electrically and thermally insulating.

The control of iridescence is a particularly desirable property; biological architectures demonstrate that it is possible to tailor the iridescence reflection from photonic architectures, often by developing optimised irregularities in the structure to varying extents. Synthetic PhCs exhibiting a FCPBG, i.e. angle-independent colour, are normally only achieved using materials with a RI contrast far higher than that found in naturally occurring systems. Quasi-ordered 3D PhCs have been discovered in the weevil Eupholus magnificus that may help to realise these aesthetics [29] by providing the prospective engineer with an additional selection of viable materials. For more extreme levels of disorder such as that found in the ultra-thin scales of Cyphochilus, approximately 5 ixm thick, the whiteness, brightness and opacity exhibited are significantly greater than that of current synthetic coatings. Thus, mimicking this exquisite whiteness has inspired research into ultra-thin optical coatings in both the paper and textiles industry [30]. Establishing control over the selfassembly processes associated with forming Bouligand structures may also provide further avenues for biomimetic applications.

\section{SUMMARY}

In this paper, we elucidate some of the principle properties of biological photonics in beetles, with particular focus on 1D multilayer and helicoidal Bouligand structures. Both these structures are iridescent, although this is not true for all structural colours in beetles. We have summarised the key governing properties of these structures and described how their physical characteristics and material specifics can influence their interaction with light. For these architectures, angle-independent colour is manifested via the variation of layer thickness and helicoidal pitch length creating a broad- 
band appearance. More broadly in beetles, a disordered distribution of scattering features on the surface of the elytron gives rise to broadband scattering, whilst quasi-ordered 2D and 3D PhCs produce narrowband angle-independent colour. Although a mechanistic, experimental approach is critical to elucidate the optical functionality of biological reflectors, it is important to relate these structures to their role within the organism. This necessitates a phylogenetic and ecological context for a more complete understanding of beetle chroma that can be implemented in the field of biomimetics.

\section{REFERENCES}

[1] Fox, D.L., Animal Biochromes and Structural Colours, University of California Press: Berkeley, CA, 1976.

[2] Srinivasarao, M., Nano-optics in the biological world: beetles, butterflies, birds, and moths. Chem Rev, 99(7), pp. 1935-1961, 1999. doi: http://dx.doi.org/10.1021/cr970080y

[3] Vukusic, P. \& Sambles, J.R., Photonic structures in biology. Nature, 424, pp. 852-855, 2003. doi: http://dx.doi.org/10.1038/nature01941

[4] Shawkey, M.D., Morehouse, N.I. \& Vukusic, P., A protean palette: colour materials and mixing in birds and butterflies. $J$ R Soc Int, 6, pp. S221-S231, 2009. doi: http://dx.doi.org/10.1098/ rsif.2008.0459.focus

[5] Mathger, L.M., Denton, E.J., Marshall, N.J. \& Hanlon, R.T., Mechanisms and behavioural functions of structural coloration in cephalopods. JR Soc Int, 6, pp. S149-S163, 2009. doi: http:// dx.doi.org/10.1098/rsif.2008.0366.focus

[6] Glover, B.J. \& Whitney, H.M., Structural colour and iridescence in plants: the poorly studied relations of pigment colour. Annals of Botany, 105(4), pp. 505-11, 2010. doi: http://dx.doi. org/10.1093/aob/mcq007

[7] Seago, A.E., Brady, P., Vigneron, J.P. \& Schultz, T.D., Gold bugs and beyond: a review of iridescence and structural colour mechanisms in beetles (Coleoptera). JR Soc Int, 6, pp. S165S184, 2009. doi: http://dx.doi.org/10.1098/rsif.2008.0354.focus

[8] Neville, A.C., Biology of the Arthropod Cuticle, Springer-Verlag: New York, 1975. doi: http:// dx.doi.org/10.1002/mmnd.4810230411

[9] Schultz, T.D. \& Rankin, M.A., Developmental changes in the interference reflectors and colorations of tiger beetles (Cicindela). J Exp Biol, 117, pp. 111-117, 1985.

[10] Yablonovitch, E., Inhibited spontaneous emission in solid-state physics and electronics. Phys Rev Lett, 58(20), pp. 2059-2062, 1987. doi: http://dx.doi.org/10.1103/physrevlett.58.2059

[11] John, S., Strong localisation of photons in certain disordered dielectric superlattices. Phys Rev Lett, 58(23), pp. 2486-2489, 1987. doi: http://dx.doi.org/10.1103/physrevlett.58.2486

[12] Joannopoulos, J.D., Johnson, S.G., Winn, J.N. \& Meade, R.D., Photonic Crystals: Moulding the Flow of Light, 2nd edn., Princeton, NJ: Princeton University Press, 2008.

[13] Born, M. \& Wolf, E., Principles of Optics, Oxford: Pergamon Press, 1980. doi: http://dx.doi. org/10.1126/science.131.3399.495

[14] Land, M.F., The physics and biology of animal reflectors. Progress in Biophysics and Molecular Biology, 24, pp. 75-106, 1972. doi: http://dx.doi.org/10.1016/0079-6107(72)90004-1

[15] Jordan, T.M., Partridge, J.C. \& Roberts, N.W., Non-polarizing broadband multilayer reflectors in fish. Nature Photonics, 6(11), pp. 759-763, 2013. doi: http://dx.doi.org/10.1038/npho$\underline{\text { ton. } 2012.260}$

[16] Neville, A.C. \& Caveney, S., Scarabaeid beetle exocuticle as an optical analogue of cholesteric liquid crystals. Biol Rev, 44(4), pp. 531-562, 1969. doi: http://dx.doi.org/10.1111/j.1469$\underline{\text { 185x.1969.tb00611.x }}$ 
[17] Starkey, T. \& Vukusic, P., Light manipulation principles in biological photonic systems. Nanophotonics, 2(4), pp. 289-307, 2013. doi: http://dx.doi.org/10.1515/nanoph-2013-0015

[18] Caveney, S., Cuticle reflectivity and optical activity in scarab beetles: the role of uric acid. Proc $R$ Soc Lond B, 178, pp. 205-225, 1971. doi: http://dx.doi.org/10.1098/rspb.1971.0062

[19] Jewell, S.A., Vukusic, P. \& Roberts, N.W., Circularly polarized colour reflection from helicoidal structures in the beetle Plusiotis boucardi. New J Phys, 9, pp. 1-10, 2007. doi: http://dx.doi. org/10.1088/1367-2630/9/4/099

[20] Sharma, V., Crne, M., Park, J.O. \& Srinivasarao, M., Structural origin of circularly polarized iridescence in jeweled beetles. Science, 325, pp. 449-451, 2009. doi: http://dx.doi.org/10.1126/ science. 1172051

[21] Arwin, H., Magnusson, R. \& Landin, J., Chirality-induced polarization effects in the cuticle of scarab beetles: 100 years after Michelson. Phil Mag, 92(12), pp. 1583-1599, 2011. doi: http:// dx.doi.org/10.1080/14786435.2011.648228

[22] Bouligand, Y., Twisted fibrous arrangements in biological materials and cholesteric mesophases. Tissue \& Cell, 4(2), pp. 189-217, 1972. doi: http://dx.doi.org/10.1016/s0040$\underline{8166(72) 80042-9}$

[23] Neville, A.C. \& Luke, B.M., Form optical activity in crustacean cuticle. J Insect Physiol, 17, pp. 519-526, 1971. doi: http://dx.doi.org/10.1016/0022-1910(71)90030-8

[24] Mathger, L.M., Rapid colour changes in multilayer reflecting stripes in the paradise whiptail, Pentapodus paradiseus. J Exp Biol, 206, pp. 3607-3613, 2003. doi: http://dx.doi.org/10.1242/ jeb.00599

[25] Giraud-Guille, M.M., Belamie, E. \& Mosser, G., Organic and mineral networks in carapaces, bones and biomimetic materials. C R Palevol, 3, pp. 503-513, 2004. doi: http://dx.doi. org/10.1016/j.crpv.2004.07.004

[26] Moogan, T., Bacterial cell wall structre. Nature Biotech, 24(4), p. 421, 2006.

[27] Parker, A.R., 515 million years of structural colour. IOP: J Opt A: Pure Appl Opt, 2, pp. R15R28, 2000. doi: http://dx.doi.org/10.1088/1464-4258/2/6/201

[28] Hegedus, R., Szel, G. \& Horvath, G., Imaging polarimetry of the circularly polarizing cuticle of scarab beetles (Coleoptera: Rutelidae, Cetoniidae). Vision Research, 46(17), pp. 2786-2797, 2006. doi: http://dx.doi.org/10.1016/j.visres.2006.02.007

[29] Pouya, C., Stavenga, D.G. \& Vukusic, P., Discovery of ordered and quasi-ordered photonic crystal structures in the scales of the beetle Eupholus magnificus. Opt Exp, 19(12), pp. 11355 11364, 2011. doi: http://dx.doi.org/10.1364/oe.19.011355

[30] Vukusic, P., Hallam, B. \& Noyes, J., Brilliant whiteness in ultrathin beetle scales. Science, 315, p. 348, 2007. doi: http://dx.doi.org/10.1126/science.1134666 